\title{
Measurement of the intersystem crossing rate in aluminum tris(8-hydroxyquinoline) and its modulation by an applied magnetic field
}

\author{
Sijie Zhang, Jingyao Song, T. Kreouzis, and W. P. Gillin ${ }^{\text {a) }}$ \\ Department of Physics, Centre for Materials Research, Queen Mary University of London, Mile End Road, \\ London, E1 4NS, United Kingdom
}

(Received 6 April 2009; accepted 14 July 2009; published online 21 August 2009)

\begin{abstract}
The rate constant for intersystem crossing in aluminum tris(8-hydroxyquinoline) was measured using the time dependence of the luminescence under high excitation intensity and modeling using a rate equation approach. Under high illumination levels intersystem crossing results in the transfer of singlets into triplets, which due to their long lifetime effectively remove molecules from participating in photoluminescence. The intersystem crossing rate was found to be $\sim 2.2$ $\times 10^{4} \mathrm{~s}^{-1}$ at $80 \mathrm{~K}$. The presence of a magnetic field was found to increase the rate constant by $\sim 10 \%$ with applied fields of $\sim 100 \mathrm{mT}$. (C) 2009 American Institute of Physics.

[DOI: $10.1063 / 1.3204015]$
\end{abstract}

\section{INTRODUCTION}

The role of magnetic fields on the optical and electrical performance of organic light emitting diodes (OLEDs) has been receiving increasing attention in the last few years due to the large increases in efficiency and current through devices that can be obtained by the application of relatively modest fields. In 2003 Kalinowski et al. ${ }^{1}$ observed that for an aluminum tris (8-hydroxyquinolate) $\left(\mathrm{Alq}_{3}\right)$ device an increase of $\sim 5 \%$ in light output could be obtained with the application of a magnetic field of $\sim 0.5 \mathrm{~T}$. This increase in the electroluminescence was attributed to the increased mixing of electron-hole pair states prior to exciton formation. In 2007 Desai et al. ${ }^{2}$ suggested that the increase in OLED efficiency they observed in $\mathrm{Alq}_{3}$ based OLEDs could be due to a similar process but they suggested that the magnetic field was acting directly on the excitons, altering the intersystem crossing rate, rather than on the pair states.

In 2001 Braun et al. ${ }^{3}$ showed that for $\mathrm{Alq}_{3}$ it was possible to obtain significant triplet populations $(\sim 25 \%)$ at low temperature $(1.3 \mathrm{~K})$ through optical excitation due to the intersystem crossing from the singlet to the triplet states. This work was extended in 2004 by Cölle and Brütting ${ }^{4}$ where they showed how it was possible to estimate the triplet concentration in the steady state caused by the intersystem crossing. In this work we have gone on to develop the rate equation model for the system and have used it to estimate the rate constant for intersystem crossing in $\mathrm{Alq}_{3}$. We have also looked at the effect of a magnetic field on the photoluminescence intensity for $\mathrm{Alq}_{3}$ and have estimated the change in the intersystem crossing rate caused by an applied magnetic field.

\section{EXPERIMENTAL METHOD}

Experiments were performed on powdered $\mathrm{Alq}_{3}$, which was purchased from Aldrich and purified by train sublima-

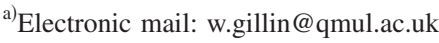

tion. Freshly purified material was placed into a continuous flow cryostat in a nitrogen ambient. Photoluminescence was excited using a $10 \mathrm{~mW} 405 \mathrm{~nm}$ laser, which was focused onto a $<0.1 \mathrm{~mm}$ spot. The laser was mechanically modulated at $5 \mathrm{~Hz}$ with a rise time of $\sim 100 \mu \mathrm{s}$. Luminescence was dispersed in a monochromator and detected using a S-20 photomultiplier or a silicon avalanche photodiode. To determine the effect of a magnet field on the photoluminescence the cryostat was placed between the poles of an electromagnet and the photoluminescence intensity was measured using conventional lock-in amplification. Measurements were made as a function of increasing magnetic field alternated with measurements at $0 \mathrm{mT}$. The two null field measurements were used to provide an average against which the effect of the magnetic field could be determined.

\section{RESULTS AND DISCUSSION}

Figure 1 shows a typical photoluminescence decay transient for $\mathrm{Alq}_{3}$ recorded at the peak of the photoluminescence spectrum at a wavelength of $520 \mathrm{~nm}$ and at sample tempera-

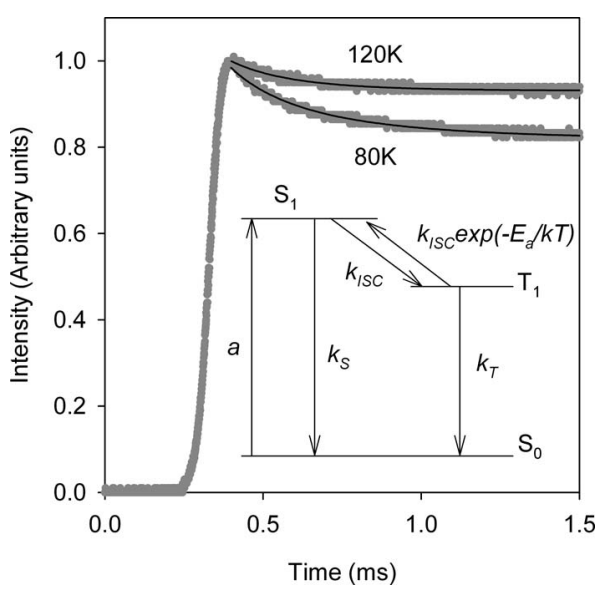

FIG. 1. Time dependence of the $520 \mathrm{~nm}$ photoluminescence from $\mathrm{Alq}_{3}$ at 80 and $120 \mathrm{~K}$. The inset shows the model used to fit the luminescence decay. 
TABLE I. Experimentally determined and fitting decay times for the photoluminescence and the percentage change in photoluminescence intensity from the initial value as a function of temperature. The parameters used for the fitting were $k_{\mathrm{ISC}}=2.2 \times 10^{4} \mathrm{~s}^{-1}, \quad a=1.4 \times 10^{8} / S_{0}$ (initial), and $E_{a}$ $=15 \mathrm{meV}$. The errors in the experimentally determined average lifetimes are $\pm 10 \%$.

\begin{tabular}{lccc}
\hline \hline & \multicolumn{3}{c}{ Temperature } \\
\cline { 2 - 4 } & $80 \mathrm{~K}$ & $100 \mathrm{~K}$ & $120 \mathrm{~K}$ \\
\hline Experimental $\langle\tau\rangle$ & $326 \mu \mathrm{s}$ & $225 \mu \mathrm{s}$ & $222 \mu \mathrm{s}$ \\
Experimental $\Delta \mathrm{I}$ & $-9.9 \%$ & $-7.3 \%$ & $-3.8 \%$ \\
Fitted $\tau$ & $335 \mu \mathrm{s}$ & $227 \mu \mathrm{s}$ & $175 \mu \mathrm{s}$ \\
Fitted $\Delta \mathrm{I}$ & $-10.1 \%$ & $-7.3 \%$ & $-5.6 \%$ \\
\hline \hline
\end{tabular}

tures of 80 and $120 \mathrm{~K}$. Photoluminescence spectra were recorded for both the prompt and steady state emission and no change in spectral shape was observed. It can be seen that the photoluminescence decay is very temperature sensitive and was difficult to observe above $120 \mathrm{~K}$ with the excitation intensity available. The rise time of the signal perfectly matches the laser intensity and is defined by the speed with which the mechanical chopper can switch the beam. The decay in the luminescence is analogous to the bleaching of laser dyes ${ }^{5}$ and has previously been attributed to intersystem crossing from the singlet to triplet states. ${ }^{3,4}$ The triplet states, being long lived, effectively remove molecules from further photoexcitation.

We have fitted the decay process with a stretched exponential function of the form,

$$
I=I_{0}+I_{1} \exp \left\lfloor-(t / \tau)^{\beta}\right\rfloor
$$

where $I_{0}$ is the steady state intensity, $I_{1}$ is the initial intensity, $\tau$ is the lifetime, and $\beta$ is the stretching function. This is an empirical fit that is widely used to fit nonsingle-exponential decay processes. Although there is no physical significance to the fit it is widely used to describe a superposition of exponential relaxation times. ${ }^{6}$ In this case it highlights the fact that the $\mathrm{Alq}_{3}$ is not homogeneous, as might be expected from a sample where there are various grain sizes, which could locally affect the exciton lifetimes. Using this stretched exponential function, it is possible to define a lifetime distribution function and calculate an average relaxation time $\langle\tau\rangle$,

$$
\langle\tau\rangle=\frac{\tau}{\beta} \Gamma\left(\frac{1}{\beta}\right),
$$

where $\Gamma()$ is the Gamma function. It is these $\langle\tau\rangle$ values that are given in Tables I-III. It should be noted that the experimental reproducibility of this data is $\pm 10 \%$.

In order to interpret this data we have a simple rate equation model (shown schematically in the inset to Fig. 1). In this model we have a pump, which populates the singlet level from the ground state and two transfer processes either back to the ground state (fluorescence) or into the triplet state through intersystem crossing. From the triplet state there is the possibility for intersystem crossing back into the singlet level, which we have made thermally activated to account for the temperature dependence observed, or there is decay of the triplet state back to the ground state. The rate equations for this system can be written as,

$$
\begin{aligned}
& \frac{d S_{0}}{d t}=k_{S} S_{1}+k_{T} T_{1}-a S_{0}, \\
& \frac{d S_{1}}{d t}=a S_{0}+k_{\mathrm{ISC}} T_{1} \exp \left(-E_{a} / k T\right)-k_{S} S_{1}-k_{\mathrm{ISC}} S_{1}, \\
& \frac{d T_{1}}{d t}=k_{\mathrm{ISC}} S_{1}-k_{T} T_{1}-k_{\mathrm{ISC}} T_{1} \exp \left(-E_{a} / k T\right),
\end{aligned}
$$

where $S_{0}, S_{1}$, and $T_{1}$ are the populations of the ground state, singlet state, and triplet state, respectively, $k_{S}$ and $k_{T}$ are the rate constants for singlet and triplet decay, $k_{\mathrm{ISC}}$ is the rate constant for intersystem crossing, $a$ is the pump rate, and $E_{a}$ is the activation energy for the triplet to singlet interchange. Literature values for the singlet lifetimes show that it varies by $<25 \%$ over the temperature range of interest. However, in the rate equation model, because the rate constant for singlet recombination is $>1000$ times greater than the next smallest term (the ISC rate), small perturbations in $k_{S}$ have no effect and hence the value was kept constant for all the modeling. A lifetime of $18 \mathrm{~ns}$ for the singlet state was taken as representative of the literature values ${ }^{7}$ and was used for all temperatures. Values for the triplet lifetime were dependent on temperature and taken from the delayed lifetime data of Ref. 8 for temperatures between 80 and $120 \mathrm{~K}$. The equations were solved numerically to determine the populations of the three levels as a function of time and the time dependence of the singlet population was compared to the lumi-

TABLE II. Experimentally determined and fitted decay times for the photoluminescence and the percentage change in photoluminescence intensity from the initial value as a function of initial intensity. The fitting parameters were the same as used in Table I. The errors in the experimentally determined average lifetimes are $\pm 10 \%$.

\begin{tabular}{lccccc}
\hline \hline & \multicolumn{5}{c}{ Percentage intensity } \\
\cline { 2 - 6 } & $100 \%$ & $79.4 \%$ & $50 \%$ & $31.6 \%$ & $10 \%$ \\
\hline Experimental $\langle\tau\rangle$ & $326 \mu \mathrm{s}$ & $358 \mu \mathrm{s}$ & $319 \mu \mathrm{s}$ & $305 \mu \mathrm{s}$ & $361 \mu \mathrm{s}$ \\
Experimental $\Delta \mathrm{I}$ & $-9.9 \%$ & $-8.2 \%$ & $-7.6 \%$ & $-5.8 \%$ & $-2.6 \%$ \\
Fitted $\tau$ & $335 \mu \mathrm{s}$ & $340 \mu \mathrm{s}$ & $352 \mu \mathrm{s}$ & $360 \mu \mathrm{s}$ & $369 \mu \mathrm{s}$ \\
Fitted $\Delta \mathrm{I}$ & $-10.1 \%$ & $-8.8 \%$ & $-5.7 \%$ & $-3.7 \%$ & $-1.2 \%$ \\
\hline \hline
\end{tabular}


TABLE III. Experimentally determined effect of magnetic field on the decay times for the photoluminescence and the percentage change in photoluminescence intensity as a function of magnetic field. The errors in the average lifetimes are $\pm 10 \%$.

\begin{tabular}{lcccccc}
\hline \hline & \multicolumn{7}{c}{ Magnetic field } \\
\cline { 2 - 7 } & $0 \mathrm{mT}$ & $55 \mathrm{mT}$ & $82 \mathrm{mT}$ & $109 \mathrm{mT}$ & $135 \mathrm{mT}$ & $162 \mathrm{mT}$ \\
\hline Experimental $\langle\tau\rangle$ & $214 \mu \mathrm{s}$ & $210 \mu \mathrm{s}$ & $198 \mu \mathrm{s}$ & $215 \mu \mathrm{s}$ & $189 \mu \mathrm{s}$ & $171 \mu \mathrm{s}$ \\
Experimental $\Delta \mathrm{I}$ & -4.3 & -4.3 & -4.7 & -4.8 & -4.3 & -4.5 \\
\hline \hline
\end{tabular}

nescence decay curve. Given that $k_{S}$ and $k_{T}$ are defined, the only variables in the model are $k_{\mathrm{ISC}}, E_{a}$, and the pump intensity $a$. The fact that the photoluminescence decay is very temperature sensitive and is not visible at temperatures greater than $120 \mathrm{~K}$ shows that the activation energy for back transfer from the triplet to the singlet state must be low. We found that a value of $15 \pm 5 \mathrm{meV}$ was needed to model the observed temperature dependence while keeping all other fitting parameters constant. Given the $\sim 0.5 \mathrm{eV}$ difference in energy between the singlet and triplet energies ${ }^{9}$ this value is very small and this will be discussed later.

Figure 2 shows the output from the rate equation model as either (a) $k_{\text {ISC }}$ is changed while keeping the pump intensity constant or (b) the pump intensity is changed while keeping $k_{\text {ISC }}$ constant. It can be seen that the values for $k_{\text {ISC }}$ in the model primarily define the decay time in the observed photoluminescence whereas the excitation intensity, $a$, determines the magnitude of the drop in photoluminescence observed. In Fig. 2(a) $k_{\text {ISC }}$ is varied from 2 to $3 \times 10^{4} \mathrm{~s}^{-1}$ and the lifetime of the decay decreases from 367 to $250 \mu$ s with little change $(<1 \%)$ in the magnitude of the steady state intensity. From Fig. 2(b) however, it can be seen that reducing the pump intensity by $90 \%$ reduces the lifetime by less than $10 \%$ but has a dramatic effect on the magnitude of the steady state intensity. In order to observe a significant drop in the $S_{1}$ population with time an excitation intensity of greater than $k_{S} / S_{0}$ (initial) is required. From Fig. 1 we have measured the average decay time and reduction in intensity of the photoluminescence as a function of temperature and the data are presented in Table I. Also given in Table I are the results from the rate equation model as a function of temperature using $E_{A}=15 \mathrm{meV}, k_{\mathrm{ISC}}=2.2 \times 10^{4} \mathrm{~s}^{-1}$, and an intensity of $1.4 \times 10^{8} / S_{0}$ (initial). This intersystem crossing rate is $\sim 100$ times greater than the triplet recombination rate at $80 \mathrm{~K}$ and $\sim 2500$ times less than the singlet recombination rate. The fact that $k_{\mathrm{ISC}}$ is so much less than $k_{S}$ means that the model is insensitive to small changes in the singlet lifetime.

In order to test the model, the laser intensity in the experiment, at $80 \mathrm{~K}$, was varied using neutral density filters to $79.4 \%, 50 \%, 31.6 \%$, and $10 \%$ of its initial value. This was found to have very little effect on the decay lifetime observed in the photoluminescence with any changes being less than the experimental reproducibility of $\sim 10 \%$. However the reduction in excitation intensity resulted in a dramatic reduction in the luminescence decay from its initial value, when the laser is first switched on. Table II gives the experimentally determined average lifetime and intensity reduction and the values obtained from the rate equation model using the same parameters as used for the modeling of the temperature dependence. It can be seen that both the lifetime and reduction in intensity show very good agreement. The effect of laser intensity should also show up any triplet-triplet annihilation (TTA) process if it were occurring in our samples, as TTA scales as the square of the triplet concentration. Our results showed no evidence of TTA (for example delayed fluorescence) and hence we did not need to include it in the rate equation model.

The only difference between the model presented here and that given by Cölle and Brütting ${ }^{4}$ is that in their work there was no intersystem crossing back from the triplet to the singlet state. We have tried to model the data by removing this process from the rate equation model. Although it is
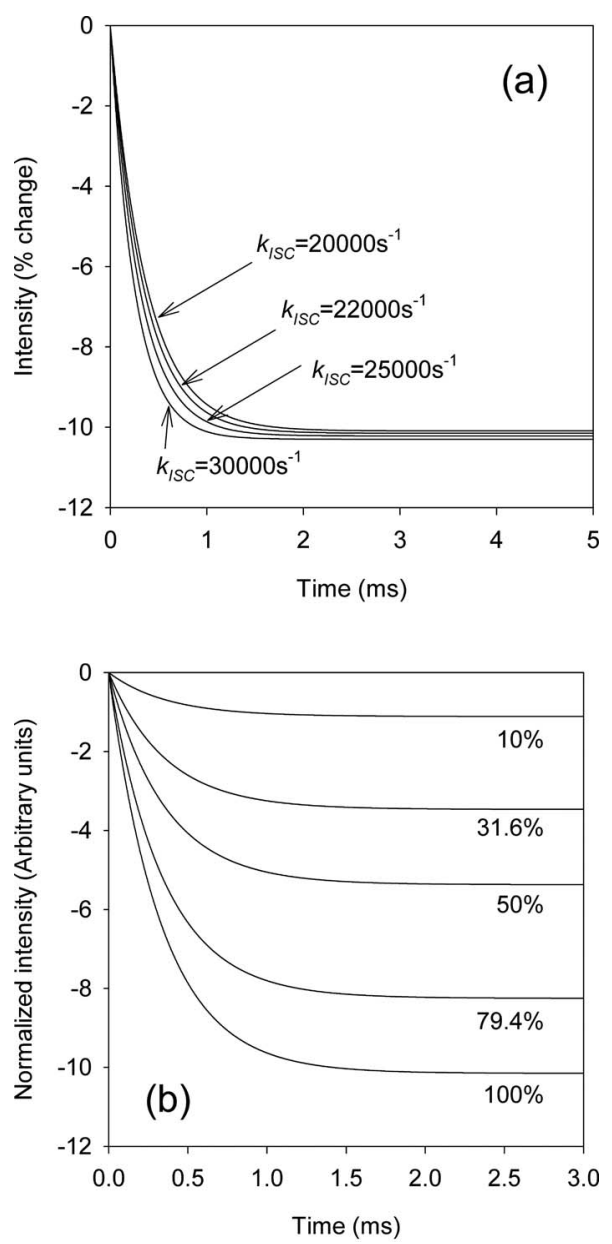

FIG. 2. The calculated time dependence of the singlet population derived from the rate equation model presented in Eq. (3). The data are presented as percentage change in intensity from the initial value. In part (a) the intensity is kept constant and $k_{\mathrm{ISC}}$ is varied while in part (b) $k_{\mathrm{ISC}}$ is kept constant and the intensity is reduced. 
possible to adjust $k_{\text {ISC }}$ and excitation intensity to model decay curves at a given temperature the effect of changing the excitation intensity by a factor of 10 is to change not just the magnitude of the luminescence decay but also to alter the decay time by an order of magnitude. This is completely contrary to the experimental results we have obtained. These results clearly show that not only does intersystem crossing occur from the singlet to the triplet state but also that it must occur in the opposite direction with very similar efficiency. The modeling we have employed has assumed a single intersystem crossing rate and activation energy. From the work of Cölle and Gärditz ${ }^{9}$ on $\mathrm{Alq}_{3}$ phosphorescence they found that the triplet lifetime was not a smooth function of temperature but had troughs, which corresponded to phase transitions. It would be expected that over a wider temperature range than we have used in our work there may well be changes in the intersystem crossing rate as well as the triplet lifetime. This would be particularly true when extending the work to room temperature where the triplet lifetime has been estimated to be $\sim 25 \mu$ s (Ref. 10), which is a factor of $\sim 200$ smaller than that at $80 \mathrm{~K}$. Such a lifetime would give a rate constant for triplet recombination, which was greater than the rate constant for intersystem crossing that we have determined at low temperatures. Despite the fact that this model is capable of fitting all the data from both the temperature and the excitation intensity dependence using a single set of parameters, which is impossible if the back transfer from the triplet state to the single state is removed, it is not at all clear what the mechanism for triplet to singlet conversion is. We have used a simple Arrhenius activation over an energy barrier in order to explain the observed temperature dependence but the measured barrier height of $15 \mathrm{meV}$ is much smaller than the $\sim 0.5 \mathrm{eV}$ energy separation between the triplet and singlet levels. What is clear from this very low activation energy is that the back transfer from the triplet to singlet is only a factor of 5-10 less than the intersystem crossing rate from the singlet to triplet state. Given the very large difference in energy a simple thermal barrier from the triplet to singlet state does not appear to be possible. One possible mechanism would be through higher excited states of the triplet. Transient state absorption spectroscopy of $\mathrm{Alq}_{3}$ has shown triplet absorption at a wavelength of $\sim 510 \mathrm{~nm},{ }^{11,12}$ which is coincident with the singlet emission wavelength of $\mathrm{Alq}_{3}$. It is therefore possible that, given the very high concentration of singlet recombination in our samples, the triplet to singlet interchange could be through an intermediate higher energy triplet state, which would overcome the problem of the energy barrier; this is illustrated in Fig. 3. In this scheme the excited state absorption (ESA) is an allowed process, as is the relaxation from the $T_{2}$ to $T_{1}$ state. This would suggest that the instantaneous population of the $T_{2}$ level would be lower than that of the $T_{1}$ level. However, exactly analogous to the singlet state the presence of intersystem crossing into the $S_{1}$ level would compete with relaxation to the $T_{1}$ level and provide a route to convert triplets to singlets. If this were to be the case then the simple Arrhenius expression that we have used in the rate equation model would only be an approximation and the true system would be more complicated. Despite this, it is clear from our data that there must be a

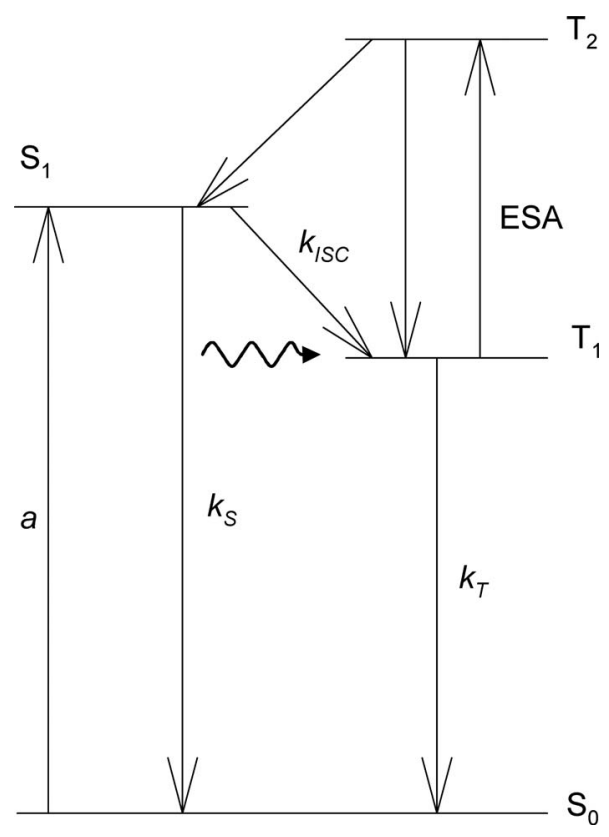

FIG. 3. A schematic of the back transfer process via ESA from the first triplet state $\left(T_{1}\right)$ to the second triplet state $\left(T_{2}\right)$. The photons for the ESA are provided by the emission from the singlet state. There may be some small temperature dependencies in these processes that would be responsible to the small activation energy term that we have in the approximated Arrhenius expression in our rate equation model.

transfer mechanism from the triplet to singlet state and which must therefore be proportional to the triplet concentration. It is this fact that the allows the simple model to provide a good fit to the data and the Arrhenius term is accounting for some small temperature dependence in some of the terms in the more complicated model. Although we could change the rate equations to account for processes in Fig. 3, at the moment they are speculative and it would make the rate equations more complicated, with more fitting terms. This would detract from the central observation that a back transfer process is essential in order to explain the results.

In order to determine the effect of a magnetic field on the intersystem crossing we repeated the photoluminescence decay measurements as a function of magnetic field, at a given intensity and temperature; the results are shown in Table III. Although the measurements with applied field showed a general reduction in the decay time there is scatter in the data and the change in the decay time is at most $\sim 10 \%$. According to our model a $\sim 10 \%$ drop in the decay time will need an $\sim 10 \%$ increase in $k_{\text {ISC }}$. We are therefore only able to say that while the effect of a magnetic field appears to be to increase $k_{\text {ISC }}$, any effect is small and from our model results is no more than a $\sim 10 \%$ change. In order to provide more comprehensive evidence of an applied magnetic field actually affecting the intersystem crossing rate we measured the photoluminescence intensity using traditional lock-in amplification at a frequency of $146 \mathrm{~Hz}$ and using a time constant of $500 \mathrm{~ms}$. The long time constant was necessary to reduce the noise in the data. To account for any drift in the sample photoluminescence intensity with time a measurement at null field was taken before and after every applied field measurement and the average of the two was used as the background 


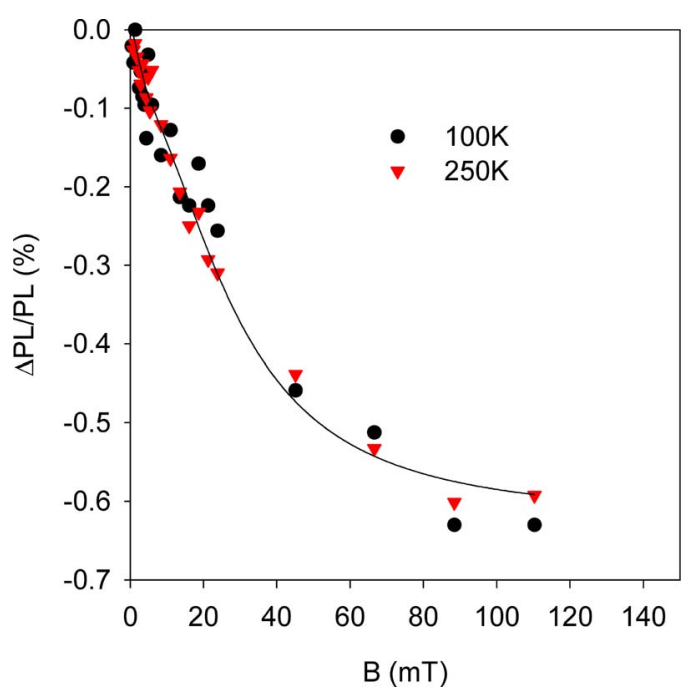

FIG. 4. (Color online) The percentage change in the PL intensity as a function of magnetic field measured using lock-in amplification at temperatures of 100 and $250 \mathrm{~K}$.

to determine the effect of the field. Using this approach Fig. 4 shows the effect of a magnetic field on the photoluminescence intensity of $\mathrm{Alq}_{3}$ as a function of magnetic field at temperatures of 100 and $250 \mathrm{~K}$. No significant effect can be seen over this temperature range.

From Fig. 2(a) it can be seen that while a change in $k_{\text {ISC }}$ did not have a large effect on the magnitude of the photoluminescence decay it does have some effect $(<1 \%)$. Using lock-in detection the signal intensity is predominantly determined by the small shift in the steady state photoluminescence intensity. From Fig. 2(a) we have determined that a $10 \%$ change in $k_{\mathrm{ISC}}$, from 2.2 to $2.5 \times 10^{4} \mathrm{~s}^{-1}$, will produce a decrease in the steady state photoluminescence intensity of $-0.67 \%$, which is almost identical to the change seen in Fig. 4. We therefore have internal consistency between our attempts to measure the changes in the decay time and the results obtained using lock-in detection and both suggest that the application of a magnetic field increases the intersystem crossing rate by $\sim 10 \%$.

The shape of the curve in Fig. 4 looks superficially similar to that seen for the effect of a B field on the efficiency of an OLED ${ }^{2,13-15}$ with a rapid change in photoluminescence intensity at low magnetic fields followed by a saturation as the field is increased. In recent work on the magnetoresistance of $\mathrm{Alq}_{3}$ based OLEDs ${ }^{2,13-15}$ we have observed that there is an increase in the efficiency of OLEDs with applied field, which is consistent with an increased singlet formation rate. We have suggested that this may be due to an increase in the intersystem crossing rate, which could result in the conversion of electrically generated triplets into singlet excitons. In electroluminescence the formation rate of triplets is three times that for singlets and hence the operational device would be expected to contain a significant triplet population. This fact is of course exploited in OLEDs by doping with phosphorescent triplet emitters. ${ }^{16}$ In photoluminescence, however, it is only singlet excitons which are primarily generated and hence an increase in the intersystem crossing rate would be expected to result in a decrease in the photoluminescence intensity as the singlets are increasingly converted into triplets.

Mermer et al. ${ }^{17}$ showed that some organic magnetoresistance data could be fitted empirically by an expression of the form $B^{2} /\left(B^{2}+B_{0}^{2}\right)$. Sheng et al. ${ }^{18}$ went on to show that this magnetic field dependence could be derived from either a hyperfine or spin-orbit ${ }^{19}$ interaction. For the efficiency of $\mathrm{Alq}_{3}$ based OLEDs we have shown that the data cannot be fitted with a single one of these curves but is very well described by two with different critical fields, ${ }^{15}$

$$
A_{1} \frac{B^{2}}{\left(B^{2}+B_{1}^{2}\right)}+A_{2} \frac{B^{2}}{\left(B^{2}+B_{2}^{2}\right)}
$$

We suggested that, as this expression would accurately fit the efficiency data but not the organic magnetoresistance data, this may be evidence that the magnetic field was acting primarily upon the intersystem crossing between the singlet and triplet states. The fact that two of these "Lorentzians" were needed to fit the data may have been evidence that there are two processes acting on the exciton. We must stress however that we have no evidence as to what the mechanism is or indeed whether these empirical fits have any physical meaning. The curve in Fig. 4 has a similar form to that obtained for the efficiency of $\mathrm{Alq}_{3}$ OLEDs and fitting the dual Lorentzian to the data produces critical field values that are very similar to those obtained in Ref. $12\left(B_{1}=3 \mathrm{mT}\right.$ and $B_{2}$ $=28 \mathrm{mT}$ for photoluminescence compared to $B_{1}=3.25 \mathrm{mT}$ and $B_{2}=36 \mathrm{mT}$ for electroluminescence). The prefactors for the two processes $\left(A_{1}\right.$ and $\left.A_{2}\right)$, however, are very different. In the efficiency data from Ref. 12 the ratio $A_{1} / A_{2}$ was $\sim 2$. In this photoluminescence data we find that ratio is $\sim 0.2$. The fact that the critical fields for the two processes are so similar suggests that the same process is responsible for the change in intersystem crossing in the two situations. However, the difference in the relative strength of the two processes indicates important differences. Throughout our work on organic magnetoresistance ${ }^{2,13-15}$ we have suggested that the effect of the magnetic field is to alter the intersystem crossing of the excitons, whereas other authors ${ }^{1,20}$ have suggested that the intersystem crossing may occur at the level of pair states before the excitons are formed. As, in this work, we would expect to be dominated by intersystem crossing at the excitonic level and it is the larger $B_{0}$ value which dominates we suggest that this may be the magnetic field dependence of the exciton intersystem crossing rate. In the electrically pumped system, where the lower $B_{0}$ values dominates, this may be the magnetic field dependence of the intersystem crossing of pair states prior to exciton formation. The fact that we have some of this process in the photoluminescence data would be evidence that there is some exciton dissociation and subsequent recombination during the photoluminescence process. However, we still are not able to state categorically what mechanism it is that is controlling the change in the intersystem crossing rate. 


\section{CONCLUSIONS}

We have shown that in $\mathrm{Alq}_{3}$ it is possible to estimate the intersystem crossing rate by modeling the time dependence of the photoluminescence under high excitation intensity. Using the model we derive an intersystem crossing rate, $k_{\mathrm{ISC}}$, of $2.2 \times 10^{4} \mathrm{~s}^{-1}$ at a temperature of $80 \mathrm{~K}$. The model also shows that intersystem crossing also occurs from the triplet to singlet state with only a relatively small energetic barrier. However, the mechanism behind transfer from the triplet to singlet state is not known.

We have also shown that a magnetic field acts to increase the intersystem crossing by $\sim 10 \%$ for fields up to $\sim 100 \mathrm{mT}$. Comparisons of this data with the effect of a magnetic field on the efficiency of an $\mathrm{Alq}_{3}$ OLED suggest that the magnetic field may affect the mixing of a pair state prior to exciton formation as well as the exciton itself.

${ }^{1}$ J. Kalinowski, M. Cocchi, D. Virgili, P. Di Marco, and V. Fattori, Chem. Phys. Lett. 380, 710 (2003).

${ }^{2}$ P. Desai, P. Shakya, T. Kreouzis, W. P. Gillin, N. A. Morley, and M. R. J. Gibbs, Phys. Rev. B 75, 094423 (2007).

${ }^{3}$ M. Braun, J. Gmeiner, M. Tzolov, M. Cölle, F. D. Meyer, W. Milius, H. Hillebrecht, O. Wendland, J. U. von Schütz, and W. Brütting, J. Chem.
Phys. 114, 9625 (2001)

${ }^{4}$ M. Cölle and W. Brütting, Phys. Status Solidi A 201, 1095 (2004).

${ }^{5}$ B. B. Snavely, Proc. IEEE 57, 1374 (1969).

${ }^{6}$ C. P. Lindsey and G. D. Patterson, J. Chem. Phys. 73, 3348 (1980).

A. D. Walser, R. Priestley, and R. Dorsinville, Synth. Met. 102, 1552 (1999).

${ }^{8}$ M. Cölle, C. Gärditz, and M. Braun, J. Appl. Phys. 96, 6133 (2004).

${ }^{9}$ M. Cölle and C. Gärditz, Appl. Phys. Lett. 84, 3160 (2004).

${ }^{10}$ M. A. Baldo and S. R. Forrest, Phys. Rev. B 62, 10958 (2000).

${ }^{11}$ R. V. Bensasson and E. J. Land, Trans. Faraday Soc. 67, 1904 (1971).

${ }^{12}$ I. Carmichael and G. L. Hug, J. Phys. Chem. Ref. Data 15, 1 (1986).

${ }^{13}$ P. Desai, P. Shakya, T. Kreouzis, and W. P. Gillin, J. Appl. Phys. 102, 073710 (2007).

${ }^{14}$ P. Shakya, P. Desai, T. Kreouzis, and W. P. Gillin, J. Appl. Phys. 103, 043706 (2008)

${ }^{15}$ P. Shakya, P. Desai, M. Somerton, G. Gannaway, T. Kreouzis, and W. P. Gillin, J. Appl. Phys. 103, 103715 (2008).

${ }^{16}$ M. A. Baldo, D. F. O’Brien, Y. You, A. Shoustikov, S. Sibley, M. E. Thompson, and S. R. Forrest, Nature (London) 395, 151 (1998).

${ }^{17}$ O. Mermer, G. Veeraraghavan, T. L. Francis, Y. Sheng, D. T. Nguyen, M. Wohlgenannt, A. Kohler, M. K. Al-Suti, and M. S. Khan, Phys. Rev. B 72, 205202 (2005).

${ }^{18}$ Y. Sheng, T. D. Nguyen, G. Veeraraghavan, O. Mermer, M. Wohlgenannt, S. Qiu, and U. Scherf, Phys. Rev. B 74, 045213 (2006).

${ }^{19}$ Y. Sheng, T. D. Nguyen, G. Veeraraghavan, O. Mermer, and M. Wohlgenannt, Phys. Rev. B 75, 035202 (2007).

${ }^{20}$ V. N. Prigodin, J. D. Bergeson, D. M. Lincoln, and A. J. Epstein, Synth. Met. 156, 757 (2006). 